\title{
Evaluation of Livestock and Poultry Farms of Different Sizes Suitable for Pollution Control Project
}

\author{
JI Yu-kun ${ }^{1, a}$, Jiang Shuai ${ }^{2, b^{*}}$, Chen Jun ${ }^{3, c}$, Liu Qiaomu ${ }^{1, d}$ \\ 1Beijing Water Consulting Corportion, Beijing, 100089 China \\ 2 Beijing Water Investment Center,Beijing,100086 China \\ 3 Beijing Institute of Water, Beijing,100048 China

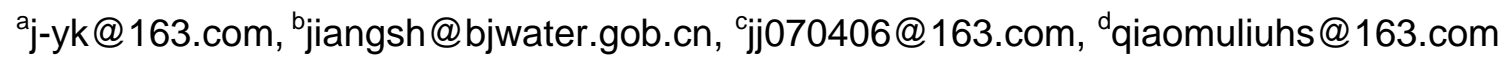

Key words: livestock breeding, biogas project, fermentation bed, aerobic composting, analytic hierarchy process, grey relational analysis

Abstract: Livestock and poultry farms in china were preliminarily divided into different scales. The popular pollution treatment projects were investigated. With the combined methods of Analytic Hierarchy Process and Grey Relational Analysis, pollution control technologies were evaluated to fit for livestock and poultry farms of different scales. Energy type biogas project is the best pollution treatment project for large-sized livestock and poultry farms, and fermentation bed ranks secondly. Deep-litter bed is the best project for medium-sized livestock and poultry farms, and energy type biogas project ranks secondly. Deep-litter bed is the best project for small-sized livestock and poultry farms, and aerobic composting ranks secondly. The evaluation method combines qualitative and quantitative evaluation, ensures that the evaluation results is scientific and impartial, and provides the livestock and poultry farms with the suitable pollution treatment technologies.

\section{Introduction}

With the intensification and large-scale development of livestock breeding industry, the scale of livestock farms Have exhibited the tendency of coexistence of traditional and modern models, from small-scale farms to large farms with tens of thousands of poultry. Poultry farms were divides into three sizes in this research: large, medium and small scale, according to the relevant provisions in the "Emission Standards for Livestock and Poultry Breeding Industry" (GB18596-2001). The method is as follows:

According to the statistics in the "Statistical Yearbook of China's Livestock" (2009), the scale of the different livestock were divided. Analysis shows that the small and medium sized livestock and poultry breeding farms (households) in China account for more than $95 \%$ of the total number.

\section{Evaluation of Livestock Pollution Control Project}

Financial status, the number of livestock and environmental requirements are primary cause to choose livestock pollution control project for different scale companies. Meanwhile, China's livestock pollution control market is mixed with good and bad technologies, and in different levels. Besides, the service condition, effect and construction fund of each technology are also different. Livestock farm pollution control technologies should be widespread evaluation, and comment on the economic, environmental and technical efficiencies and their sustainability in order to determine which pollution control technology suit for the scale of livestock and poultry enterprises. We can promote the development of the industry and make enterprises use the most suitable project through 
evaluation. This will promote China's development of livestock breeding technology and give full use of each pollution control technologies.

The research is an objective and reasonable scientific evaluation upon the existing pollution control projects for livestock and poultry farming based on such considerations. The evaluation, referring to the pollution control technologies for livestock and poultry farming recommended by the Technical Guide to the Pollution Control of the Agricultural Solid Waste (HJ588-2010), issued by China's State Environmental Protection Administration in 2010. Four pollution control technologies for livestock and poultry farming as evaluation objects: resource-based biogas project, environment-friendly biogas project, fermentation bed technology and aerobic composting technology. The first two projects are distinguished from each other by how to deal with the biogas slurry and residue in the final stage of the biogas project. The project that the slurry and residue is made fertilizers to the crop or processed into organic fertilizer for sale is resource-based biogas project, while the one that meets the biogas emission standards finally through further processing is considered as environment-friendly biogas project.

Analytic Hierarchy Process and Grey Relational Analysis (AHP-GRAY) evaluation method was adopted in the evaluation. Through the following steps, the advantages and disadvantages of the four pollution control technologies are concluded. 1. Establish indicators levels through AHP method; 2. Establish the structure matrix by experts' scoring; 3. Determine the weights of each evaluation indicator of the pollution control project for livestock breeding; 4. Adopt the gray relational analysis to calculate correlation between each pollution control technology and its ideal state. This gives full play of expert's professional knowledge, while avoiding the deviation caused by the qualitative evaluation. By the calculation method for correlation in the gray relational analysis, the qualitative and quantitative evaluations are combined.

\section{Technical Evaluation of Livestock and Poultry Farms with Different Sizes}

This evaluation adopts a combination of AHP and GRAY to reduce the impact of human factors on the results, and to avoid deviation caused by qualitative evaluation. The combination of quantitative and qualitative approaches ensures an objective, scientific and reasonable result.

\subsection{Analytic Hierarchy Process}

AHP[5] is a systematization, modeling and quantification approach to decision thinking of complicated phenomenon[6], proposed in the1970s by T. L. Saaty, an U.S. scholar in operational research filed. The basic steps of AHP includes:

(1) Establishment of hierarchy model. The final target of the pollution prevention and control project was regarded as the evaluation object. In accordance with the systematic, applicable, independent and a combination of qualitative and quantitative principles, the indicator system which is representative, accessible and easy to quantify was selected.

The indicator evaluation system of the livestock pollution control program sets the overall goal as the improvement of environment quality. It takes the economic, environmental and technical benefits, and their sustainability as the technical evaluation criteria for the pollution control program. Specific evaluation indicators are listed under the criteria, thus forms a indicator system. 


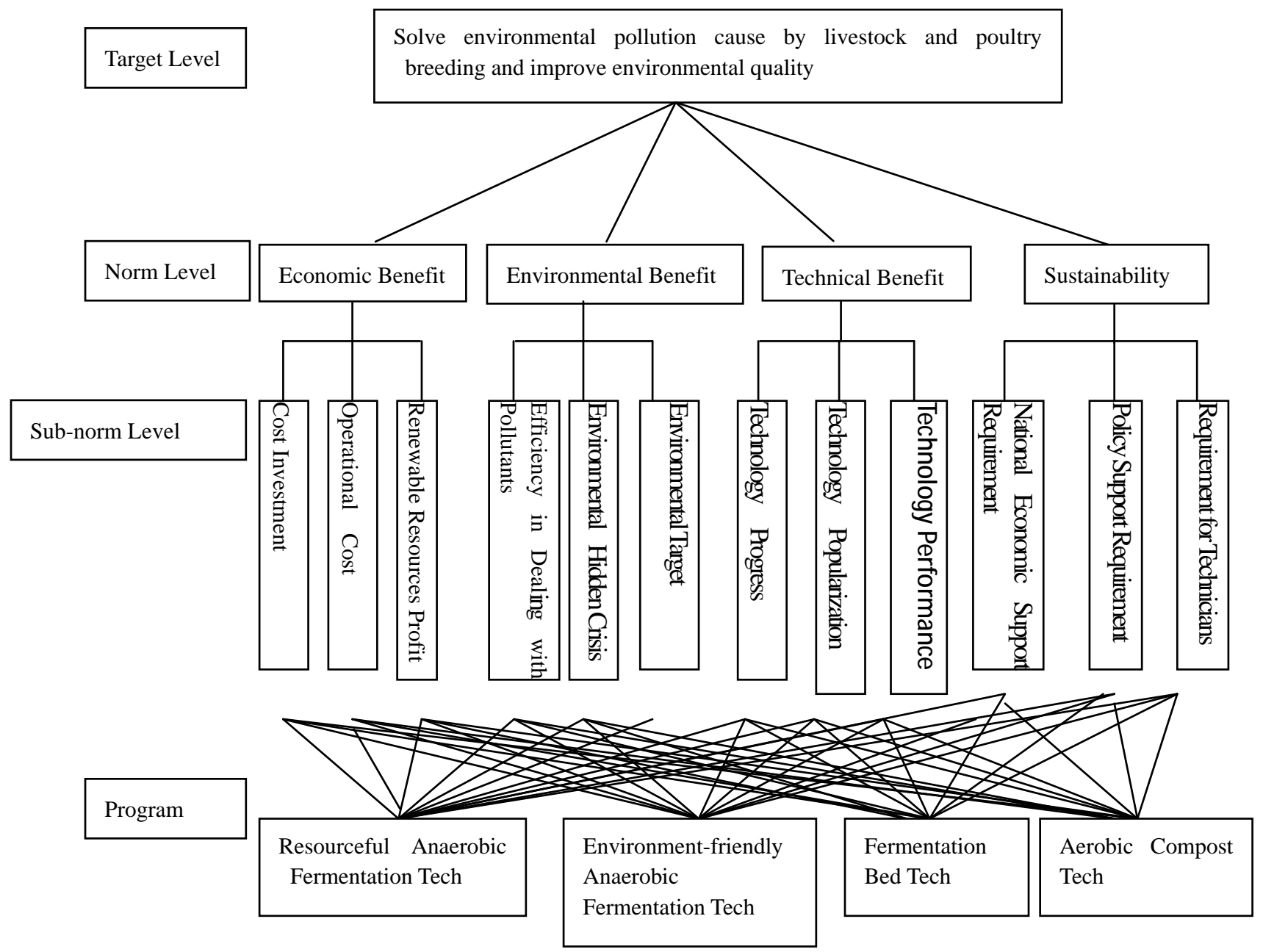

Figurer 1 Technical Hierarchy Structure of Livestock and Poultry Pollution Control

(2) Form the judgment matrix. Hire the senior experts to use 1-9 and the reciprocal scaling method to compare each two and form the judgment matrix.

(3) Check the single ranking of hierarchy and its consistency. Calculate the latent root and eigenvectors of the judgment matrix. Through normalization, the ranking weight with the relative importance between the elements in the same hierarchy and the corresponding factors in another hierarchy. The commonly used calculating method:

(4) Order of the hierarchy and its consistency test. From the top to the lowest, check the superposition weights for each hierarchy, and obtain the weights ranking that lowest is relative to that of the top. Calculate the ratio RI of consistency indicator CI in single order with its corresponding average random consistency indicator $\mathrm{CR}$,

\section{Results}

(1) The following applicability of different techniques is obtained by determining the optimal indicator list of twelve indicators of the livestock and poultry industry with different sizes, and using grey relation analysis to calculate the correlation between the indicator list of different pollution control project and the best indicator. 
Table 1 list of pollution control technology for livestock and poultry breeding industry of different sizes

\begin{tabular}{lcccccc}
\hline \multirow{2}{*}{ Types } & Large Size & \multicolumn{3}{c}{ Medium Size } & \multicolumn{2}{c}{ Small Size } \\
\cline { 2 - 7 } & Corelation & $\begin{array}{l}\text { Overall } \\
\text { Ranking }\end{array}$ & Corelation & Overall Ranking & Corelation & $\begin{array}{l}\text { Overall } \\
\text { Ranking }\end{array}$ \\
\hline \multicolumn{1}{c}{$\begin{array}{l}\text { Energy Biogas Project } \\
\text { Environment-friendly Biogas }\end{array}$} & 0.37 & 1 & 0.25 & 2 & 0.23 & 3 \\
Project & 0.18 & 3 & 0.10 & 4 & 0.11 & 4 \\
Fermentation Bed & 0.31 & 2 & 0.42 & 1 & 0.46 & 1 \\
Organic Compost & 0.14 & 4 & 0.24 & 3 & 0.24 & 2 \\
\hline
\end{tabular}

Livestock and poultry industry of different sizes have different requirements for pollution treatment projects because of different economic status, opinion for environment and scale. Combined with expert's evaluation results and weights of each indicator through AHP, expert's subjectivity is avoided. Meanwhile, by GRAY, qualitative analysis is transformed into quantitative one. Therefore, the results are more objective, impartial and to the actual situation. This evaluation provides a good reference for the livestock industry to choose the appropriate pollution control technology.

\section{Conclusions}

1) Supporting resource-based biogas project of large-scale livestock and poultry farm. Biogas can be used as energy by villager or produce electricity to its grid for it's high yield and stable. Biogas slurry and biogas residue can owner-occupied or be sold as organic fertilizer though machines.Such as: The daily output biogas is about 3 million cubic metres of Minhe LTD's big-scale biogas anaerobic fermentation engineering which can be used to produce electricity. electric energy production is 7million KWH. Biogas slurry is used by garden and cropland around farm as organic fertilizer which make balance of manure and farmland load in ture. This company signed in CDM with the united nations in 2009, and realized benefit of greenhouse gas emission reduction.

2) Fermenting bed and compost should be supported to build in small and medium size livestock and poultry farm. Biogas project need great number construction cost at initial investment, and it's operating cost every year is high. Fermenting bed and compost don't exist those problems, and have obvious environmental effect. Fermenting bed increase profit of breed aquatics. Fujian putian youlike farming develop co's added net worth is about 6 RMB per pig which gained from raise pigs though fermenting bed.

\section{References:}

[1] Wang Kaijun. Livestock Pollution Prevention Technology and Policy [M], Beijing: Chemical Industry Press.2004.

[2] Sheng Qingkai, Wu Ying etc, Advantages of Fermentation Bed Pig-breeding Technology and its Application Problem, Environmental control.

[3] Japanese Breeding Techniques of Fermentation Bed, Contemporary Pig-breeding [J].2004,2:28-29

[4] Zeng Chu, Zeng Huanming. On the Problem of Fermentation Bed Breeding, Hunan Feed [J].2009,6:41 
[5] Chinese The Editorial Board of The Livestock Husbandry Yearbook: Chinese Livestock Husbandry Statistical Yearbook (2008), Peking, Chinese Agriculture, Book Concern(2015) [6] National Bureau of Statistics, Ministry of Environmental Protection:China Statistical Yearbook on Environment. Peking. China Statistics Concern (2009) 